\title{
The impact of ureteral Double-J stent insertion following ureterorenoscopy in patients with ureteral stones accompanied by perirenal fat stranding
}

\author{
Ercan Ogreden ${ }^{1}$, Ural Oguz $^{1}$, Erhan Demirelli ${ }^{1}$, Erdal Benli $^{2}$, Özkan Özen ${ }^{3}$ \\ ${ }^{1}$ Giresun University, Faculty of Medicine, Department of Urology, Giresun, Turkey; \\ ${ }^{2}$ Ordu University, Faculty of Medicine, Department of Urology, Ordu, Turkey; \\ ${ }^{3}$ Giresun University, Faculty of Medicine, Department of Radiology, Giresun, Turkey.
}

\begin{abstract}
Summary Objective: To evaluate the impact of ureteral stent insertion following semirigid

ureterorenoscopy (URS) in patients with perirenal fat stranding (PFS) due to ureteral stones.

Material and methods: Data of 600 patients who underwent URS were analyzed retrospectively. Seventy-two patients detected to have PFS accompanying ureteral stone were included. Patients who did not undergo double J (DJ) stent insertion following semirigid URS were classified as Group I (n: 52), while those who underwent stent insertion were classified as Group II (n: 20). Side distribution; localization of the stones, stone size, presence of fever, urinary tract infection (UTIs) and urosepsis rates were compared in the two groups.

Results: The average age of the patients was 44.4 (20-71) years. Male/female ratio and side of the stone location showed similar distribution in both groups $(p>0.05)$. Fever occurred in 23 cases (44.2\%) in Group I and in 15 cases (75\%) in Group II ( $p=0.038)$. UTIs occurred in 15 cases $(28.9 \%)$ in Group I and in 12 cases $(60 \%)$ in Group II ( $p=0.03)$. Urosepsis presented in $3(5.8 \%)$ and $5(25 \%)$ of the patients in Group I and II, respectively $(p=0.033)$.

Conclusions: According to our results, ureteral DJ stent insertion following URS in patients with PFS due to ureteral stone caused an increase on postoperative infection related complications.
\end{abstract}

KEY WORDS: Perirenal fat stranding; Ureteral stents; Ureteral stones; Ureterorenoscopy.

Submitted 15 October 2017; 10 December 2017

\section{INTRODUCTION}

Ureteral stones can lead to partial or complete obstruction of ureteral lumen (1). Computerized tomography (CT) is the ideal method for detecting obstructing stones. There are primary and secondary findings of ureteral obstruction due to stones on CT. The primary finding is the detection of stone. Secondary findings are hydronephrosis, enlarged ureter, perirenal fat stranding (PFS), pararenal facial thickening, perirenal fluid collection (2). PFS is a CT imaging of the perirenal fat tissue. Asymmetric or unilateral PFS is an important indicator of renal inflammation or acute obstruction. It is detected especially in the presence of inflammation such as acute pyelonephritis and acute obstruction secondary to ureteric stones (3). Extracorporeal Shock Wave Lithotripsy (ESWL), ureterorenoscopy (URS) and endoscopic lithotripsy are the most common treatment modalities currently used in ureteral stones. The ureteral DJ stent insertion indications are the complications that develop secondary to the presence of the stones and the complications that arise during the surgical procedure (4). However, the use of stents can lead to side effects such as pain, urinary infection, and irritable voiding symptoms $(5,6)$. Thus, we aimed to evaluate the correlation between ureteral DJ stent and infective complications such as fever, UTIs and urosepsis in patients with PFS who develop secondary to ureteral stones.

\section{MATERIAL AND METHOdS}

Data of 600 patients who underwent URS in two tertiary centers between May 2010 and May 2017 were analyzed retrospectively. Routine laboratory, complete urinalysis, urine cultures, blood cultures and CT scan results were obtained by a comprehensive review of medical records. Vital signs were also reviewed and presence of any UTIs, fever and urosepsis were noted. Urine cultures were obtained from patients with asymptomatic bacteriuria and appropriate empirical treatment was started.

Symptomatic urinary infection criteria included fever, costovertebral angle sensitivity, pyuria ( $\geq 10$ white blood cells per high-power field), and positive urine culture ( $\geq 105$ colony-forming units of uropathogen $/ \mathrm{mL}$ ).

Urosepsis criteria included at least 2 findings of Systemic Inflammatory Response Syndrome (SIRs) in the presence of infection. SIRs criteria included fever $>38 \mathrm{C}^{\circ}$ or $<36 \mathrm{C}^{\circ}$, heart rate $>90$ beats $/ \mathrm{min}$, respiratory rate $>20 / \mathrm{min}$ or $\mathrm{PaCO} 2<32 \mathrm{mmHg}$, leucocytes $>12.000 / \mathrm{mm}^{3}$ or $<$ $4.000 / \mathrm{mm}^{3}$. Patients diagnosed with urosepsis was treated empirically considering antibiotic susceptibility results. Of 600 patients, 72 with PFS and hydronephrosis due to ureteral stones were included in the study. PFS defines the appearance of edema of the fat of the perirenal space at CT. Presence of PFS, stone size, side distribution and localization of the stones were documented by reviewing the CT scans (Figure 1). Patients who did not undergo stent insertion following semirigid URS were classified as 


\section{Figure 1.}

Unenhanced tomography of a 53-year-old male patient with left lateral and left testicular pain.

[1a - perirenal fat stranding (shown by the white arrow) image, $1 b$ - perirenal lines (shown by the white arrow) and two stones in the lower calyx of left kidney (indicated by a white arrowhead). 1c - perirenal streaks (shown by the white arrow) and dilatation of the left ureter (indicated by a white arrowhead), $1 d$ - the left distally ureteral stone (shown by the white arrow)].

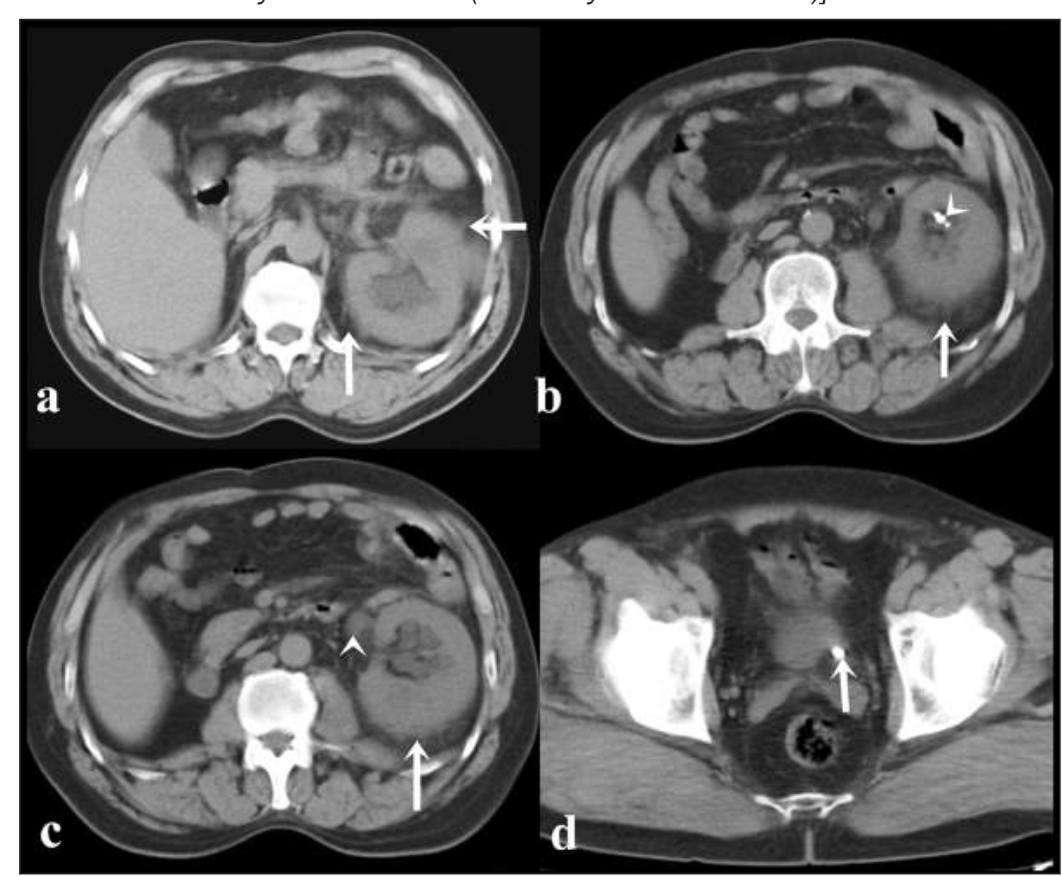

\section{Statistical analysis}

Results are presented as frequency and percentage (\%).

The abnormal distribution of data from each group was confirmed with the Kolmogorov-Smirnov test, thus statistical comparisons were performed using Mann Whitney-U Test. Chi-square test was used to examine the dependency between the groups. SPSS 22.0 software for Windows (SPSS Inc., Chicago, IL) was used for analysis of data. A P value less than 0.05 was considered statistically significant.

\section{RESULTS}

The mean age of the patients was 44 (23 70) years in Group I and 45.3 (20-71) years in Group II ( $p=0.811$ ) (Table 2). Female patients were 14 (26.9\%) and 2 (10\%) in Group I and II, respectively. Male patients were $38(73.1 \%)$ and 18 (90\%) in Group I and II, respectively $(\mathrm{p}=0.205)$ (Table 1).

Stones were detected in the right ureter in $27(51.9 \%)$ patients in Group I and 10(50\%) patients in Group II.

Left ureteric stones were detected in 25 (48.1\%) patients and 10 (50\%) patients in Group I and II, respectively $(\mathrm{p}=1.00)$ (Table 1).

Group I (n:52), while those who underwent stent insertion were classified as Group II (n:20).

Side distribution; localization of the stones, stone size, presence of fever, UTI and urosepsis rates were compared in the two groups.
Lower ureteric stones were found in 46 (88.5\%) patients in Group I and 9 (45\%) patients in Group II. Mid ureteric stones were found in $9(11.5 \%)$ patients and 6 (30\%) patients in Group I and Group II, respectively. Upper ureteral stones were observed in only 5 (25\%)

Table 1.

Demographic distribution of infective complications, gender, stone side and localization according to groups.

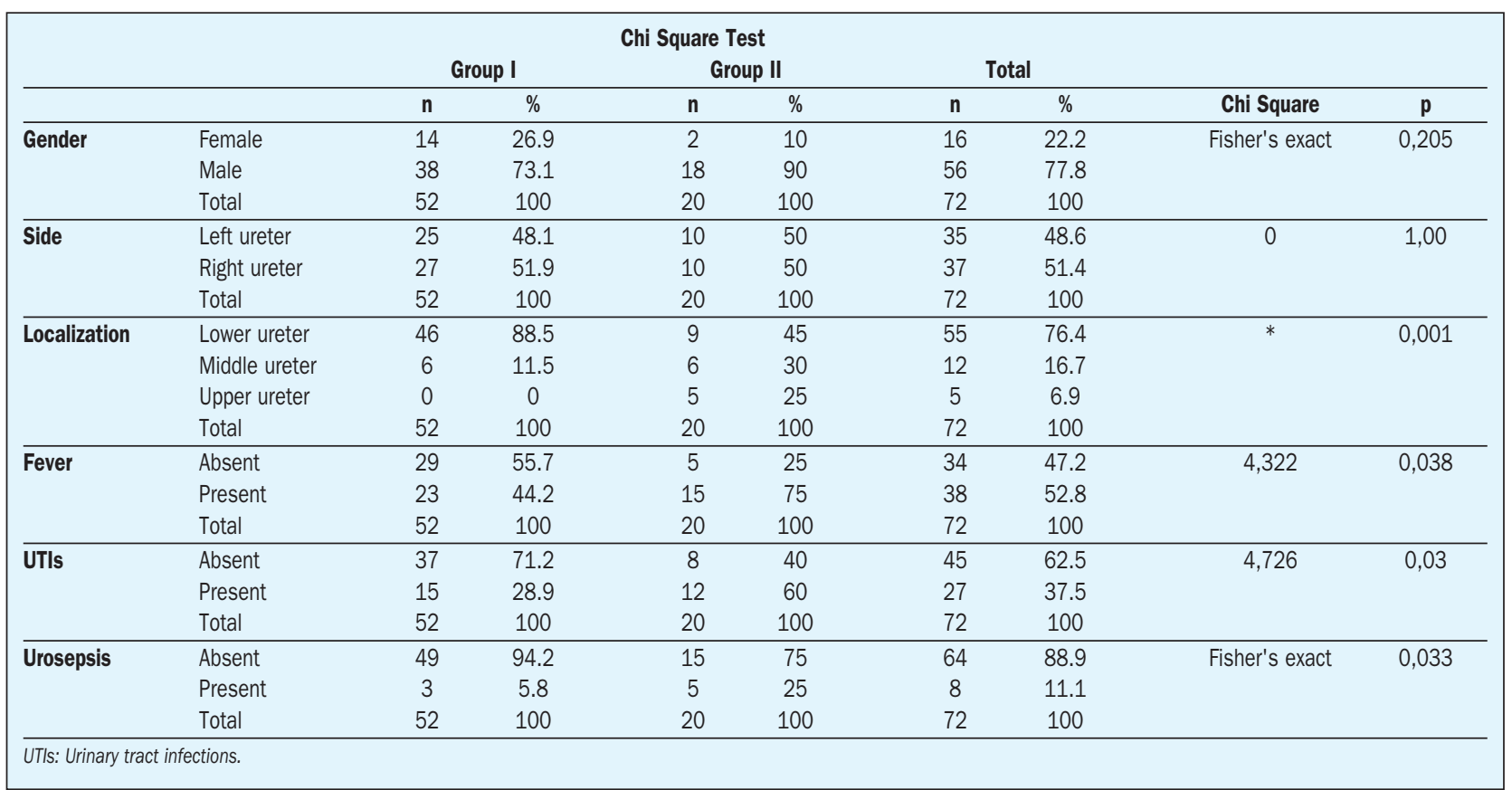


Table 2.

The differences between groups in terms of age and stone size.

\begin{tabular}{|c|c|c|c|c|c|c|c|c|c|c|}
\hline & & & & & & & & \multicolumn{3}{|c|}{ Mann Whitney U Test } \\
\hline & & $n$ & Mean & Median & Min & Max & ss & Rank Avg. & $z$ & $p$ \\
\hline \multirow[t]{3}{*}{ Age } & Group I & 52 & 44.0 & 42 & 23 & 70 & 11.9 & 36.13 & -0.239 & 0.811 \\
\hline & Group II & 20 & 45.3 & 43 & 20 & 71 & 15.1 & 37.45 & & \\
\hline & Total & 72 & 44.4 & 43 & 20 & 71 & 12.8 & & & \\
\hline \multirow[t]{3}{*}{ Size $/ \mathrm{mm}$} & Group I & 52 & 7.2 & 7 & 4 & 13 & 2.1 & 29.87 & -4.381 & 0.001 \\
\hline & Group II & 20 & 11.4 & 10 & 4 & 20 & 4.2 & 53.75 & & \\
\hline & Total & 72 & 8.3 & 8 & 4 & 20 & 3.4 & & & \\
\hline
\end{tabular}

patients in Group II ( $\mathrm{p}=0.001)$ (Table 1). Fever was detected in 23 (44.2\%) patients and $15(75 \%)$ patients in Group I and Group II, respectively ( $p=0.038$ ). In 25 of the 38 patients with fever, at least one species of a microorganism was isolated in their urine and/or blood cultures. The most isolated microorganism was E. coli (95\%).

UTI was detected in 15 patients (28.9\%) in Group I and 12 patients (60\%) in Group II ( $p=0.03)$. In 10 of the 27 patients, urine culture was positive. The most frequently isolated bacteria was E. coli $(98 \%)$.

Urosepsis was seen in $3(5.8 \%)$ patients in Group I and in $5(25 \%)$ patients in Group II $(p=0.033)$. All of these patients were found to have microorganisms in both urine and blood cultures (Table 1).

Mean size of the stones was $7.2 \mathrm{~mm}(4-13 \mathrm{~mm})$ in Group I and $11.4 \mathrm{~mm}(4-20 \mathrm{~mm})$ in Group II. Mean size of the stones did significantly differ between the groups ( $\mathrm{p}=$ 0.001) (Table 2).

Preoperative hydronephrosis was seen in all patients in both groups, whereas postoperative hydronephrosis was not seen in any patient. Success was defined as the stone free and success rates of the treatment was 100\% in both groups. Catastrophic ureteral injuries such as avulsion or perforation did not occur in any patient.

\section{Discussion}

Perirenal fat stranding (PFS) indicates the appearance of edema in the fat of the perirenal space in CT. Asymmetric or unilateral PFS is an important indicator of renal inflammation or acute obstruction. CT is the ideal method for detecting obstructing stones. There are primary and secondary findings of ureteral stones that have acute ureteral obstruction in CT. Primary findings are the appearance of stone. Secondary findings include hydronephrosis, enlarged ureter, PFS, pararenal fascia thickening, perirenal fluid collection. Secondary findings have a high positive and negative predictive value for ureteral stone presence or absence $(2,3,7)$.

Semirigid ureterorenoscopy is a rather effective and minimally invasive method of treatment for ureteral stones. Until recently, DJ stent insertion was performed in all patients who underwent URS in order to decrease the risk of postoperative ureteral edema and obstruction, to avoid the development of ureteral stenosis, to facilitate the spontaneous passage of small stone fragments and to diminish the postoperative risk of pain. However, rou- tine insertion of DJ stent has been questioned because redesigned endoscopic equipments cause less URS complications, intracorporeal lithotripsy devices are quite improved and irritative voiding symptoms secondary to DJ stent along with side effects such as hematuria, catheter migration, fever and urinary infection may be seen (8). While ureteral DJ stent insertion is not recommended in patients who do not have complications, it is still recommended in patients with complications such as mucosal edema, mucosal damage, hemorrhage, ureteral laceration and stone migration, and in patients with solitary kidney $(9,10)$. Boridy et al. (11) found that there was a significant relationship between the degree of PFS and obstruction in the study of patients with acute ureteral obstruction and the degree of obstruction was high in this study when PFS was excessive. In our study, complications related to infection in the postoperative period were found to be high in patients with PFS. PFS, urine leakage due to small tears in the calyx fornixes is seen as a linear increase in perinephric fat tissue density. However, PFS is not a specific finding. PFS is also seen in acute pyelonephritis, pyelonephrosis and renal vein thrombosis (12). It is recommended to perform contrastenhanced CT scan to exclude other possible causes in patients without stone in CT (13).

Both obstruction and stasis in ureteral lumen caused by ureteral stones and DJ catheter insertion following treatment increase the risk of urinary infection. Risk of hydronephrosis, risk of PFS, level of thickening in the pararenal fascias and level of unilateral parenchymal thickening are proportional to severity of ureteral obstruction. PFS is more common in ureteral stones complicated by infection $(14,15)$.

Stent insertion is an effective method to provide acute drainage of the hydronephrotic or pyonephrotic kidney (16). But in contrast, it may be the source of the infection in the long term period. Several studies reported bacterial colonization rates from $44 \%$ to $69 \%$ on ureteral stents and bacteriuria rates from $21 \%$ to $29.9 \%$. Mild fever, urinary tract infection, even sepsis can be seen due to bacterial colonization of DJ stents (17). In a study conducted with 87 patients who underwent DJ stent insertion following emergency intervention (n:34) or elective intervention (n:53), postoperative fever was seen in 22 (25\%) patients who did not have preoperative fever. Fever was seen in $56 \%$ of the patients who underwent stent insertion following emergency intervention, while it was present in only $6 \%$ of the patients underwent stent 
insertion following elective intervention. Hence it was reported that stent insertion following emergency intervention significantly increased risk of fever (18). In another study, DJ stent was inserted in 26 of the 48 patients who underwent URS because of distal ureteral stone and 22 patients were followed up without a stent. Urosepsis was found in only 1 patient who underwent DJ stent insertion and the difference was not significant (19). A similar study reported that presence of fever was not associated with stent insertion (20). Ibrahim at al. (21) analyzed 110 patients with stent and 110 without stent in their large series, prospectively. Fever developed in $8(7.3 \%)$ patients and UTIs developed in $5(4.5 \%)$ patients in the stent group, while fever was present in 10 (9.1\%) cases and UTIs were present in 7 (6.4\%) cases in not stented group. Although there was no significant difference between the groups, presence of fever and infection were slightly higher in not stented group. They did not evaluate the radiological findings such as PFS.

At the end of the URS procedures, stent insertion is not recommended in patients with hydronephrosis and PFS caused by ureteral stones. And the effects of the PFS on postoperative complications is still unclear. To the best of our knowledge, this is the first study focus on this topic in the literature. Compared with the literature, we found higher rates of infection related complications of URS in our study. Thus PFS due to ureteral obstruction can be a predisposing factor for the postoperative complications associated with infection. In addition, stent insertion did not reduce the risk for fever, UTIs and sepsis. Eventually, they were higher in stented patients without a significant difference.

Prior studies reported that the necessity of DJ stent insertion increases in patients with higher stone burden and in male patients $(22,23)$. In the population of this study, female/male ratio, side and mean operative times did not significantly differ between the groups ( $p>0.05)$. In contrast, mean stone burden and the localization of the stone were significantly higher in stenting patients. The surgeons' decision for inserting ureteral DJ stent seems to be affected mainly by the stone burden and the localization of the stone just like in the literature.

We acknowledge that there were several limitations of this study. The most important limitation was that the study was designed in a retrospective nature. Thus patients were not randomized, and the surgeons might not be aware of the PFS, especially when the radiologist did not report it. In addition real incidences of significant or insignificant mucosal injuries in the study groups are not clear. Therefore, we cannot present the real indications for the ureteral DJ stent insertion. Hence, we believe that our findings need to be confirmed by further randomized prospective studies.

Despite the shortcomings mentioned above, this is an important study since there is no previous data in the literature about this topic.

\section{Conclusions}

Compared with the literature, we found higher rates of infection related complications of URS in this study. Ureteral DJ stent insertion following URS due to ureteral stones did have a significant effect on postoperative infection related complications such as fever, UTIs and sepsis in patients with PFS.

\section{REFERENCES}

1. Straub M, Strohmaier WL, Berg W, et al. Diagnosis and metaphylaxis of stone disease. Consensus concept of the National Working Committee on Stone Disease for the Upcoming German Urolithiasis Guideline. World J Urol. 2005; 23:309-23.

2. Takahashi N, Kawashima A, Ernst RD, et al. Ureterolithiasis: can clinical outcome be predicted with unenhanced helical CT? Radiology. 1998; 208:97-102.

3. Stunell H, Buckley O, Feeney J, et al. Imaging of acute pyelonephritis in the adult. Eur Radiol. 2007; 17:1820-8.

4. Nabi G, CookJ, N'Dow J, McClinton S. Outcomes of stenting after uncomplicated ureteroscopy: systematic review and meta-analysis. BMJ. 2007; 334:572

5. Joshi HB, Newns N, Stainthorpe A, et al. Ureteral stent symptom questionnaire: development and validation of a multidimensional quality of life measure. J Urol. 2003; 169:1060-64.

6. Keeley Jr FX, Timoney AG. Routine stenting after ureteroscopy: think again. Eur Urol. 2007; 52:642-44.

7. Fielding JR, Steele G, Fox LA, et al. Spiral computerized tomography in the evaluation of acute flank pain: a replacement for excretory urography. J Urol. 1997; 157:2071-73.

8. Hiller N1, Berkovitz N, Lubashevsky N, et al. The relationship between ureteral stone characteristics and secondary signs in renal colic. Clinical Imaging. 2012; 36:768-72.

9. Kara C, Bayındır M, Çiçekbilek I, et al. Comparison between ureteroscopy and extracorporeal shock wave lithotripsy in the treatment of distal ureteral stones. Turkish Journal of Urology. 2009; $35: 28-33$

10. Cevik I, Dillioglugil O, Akdas A, Siegel Y. Is stent placement necessary after uncomplicated ureteroscopy for removal of impacted ureteral stones? J Endourol. 2010; 24:1263-67.

11. Boridy IC, Kawashima A, Goldman SM, Sandler CM. Acute ureterolithiasis: nonenhanced helical CT findings of perinephric edema for prediction of degree of ureteral obstruction. Radiology. 1999; 213:663-7.

12. Heneghan JP, Dalrymple NC, Verga M, et al. Soft-tissue "rim" sign in the diagnosis of ureteral calculi with use of unenhanced helical CT. Radiology. 1997; 202:709-11.

13. Chong WK, Wysoki M, Heller LG, Zegel HG. Renal carcinoma presenting with flank pain: a potential drawback of unenhanced CT. AJR Am J Roentgenol. 2000; 174:667-9.

14. Smith RC, Levine J, Rosenfeld AT. Helical CT of urinary tract stones. Radiol Clin N Am. 1999; 37:911-52.

15. Tenke P, Jackel M, Nagy E. Prevention and treatment of catheterassociated infections: Myth or reality? Eur Urol. 2004; 106-15.

16. Goldsmith ZG, Oredein-McCoy O, Gerber L, et al. Emergent ureteric stent vs percutaneous nephrostomy for obstructive urolithiasis with sepsis: patterns of use and outcomes from a 15-year experience. BJU Int. 2013; 112:122-8.

17. Paick SH, Park HK, Oh SJ, Kim HH. Characteristics of bacterial colonization and urinary tract infection after indwelling of double-j ureteral stent. Urology. 2003; 62:214-17.

18. Paz A, Amiel GE, Pick N, et al. Febrile complications follow- 
ing insertion of 100 double-J ureteral stents. J Endourol. 2005; 19:147-50

19. Srivastava A, Gupta R, Kumar A, Kapoor R, Mandhani A. Routine stenting after ureteroscopy for distal ureteral calculi is unnecessary: results of a randomized controlled trial. J Endourol. 2003; 17:871-74.

20. Cevik I, Dillioglugil O, Akdas A, Siegel Y. Is stent placement necessary after uncomplicated ureteroscopy for removal of impacted ureteral stones? J Endourol. 2010; 24:1263-67.
21. Ibrahim HM, Al-Kandari AM, Shaaban HS, et al. Role of ureteral stenting after uncomplicated ureteroscopy for distal ureteral stones: a randomized, controlled trial. J Urol. 2008; 180:961-65.

22. Matani YS, Al-Ghazo MA, Al-azab RS, et al. Emergency double-J stent insertion following uncomplicated Ureteroscopy: risk-factor analysis and recommendations. Int Braz J Urol. 2013; 39:203-8.

23. Tanriverdi $O$, Yencilek F, Koyuncu $H$, et al. Emergent stenting after uncomplicated ureteroscopy: evaluation of 23 patients. Urology. 2011; 77:305-08.

\section{Correspondence}

Ercan Ogreden, MD (Corresponding Author)

ercanogreden@gmail.com

Ural Oğuz, MD

uraloguz@yahoo.com

Erhan Demirelli, MD

erhandemirelli@yahoo.com

Giresun University, Faculty of Medicine, Department of Urology

Giresun, Turkey

Erdal Benli, MD

drerdalbenli@gmail.com

Ordu University, Faculty of Medicine, Department of Urology

Ordu, Turkey

Özkan Özen, MD

ozen@doctor.com

Giresun University, Faculty of Medicine, Department of Radiology

Giresun, Turkey 